\title{
A Protocol for Recording Visual and Motor Behaviour for Scientific Support in the Teaching and Training of Sailing in a Simulator
}

\author{
Aarón Manzanares, Francisco Segado and Ruperto Menayo \\ (Faculty of Sport, San Antonio Catholic University, Campus de Los Jerónimos, s/n \\ 30107, Guadalupe, Murcia, Spain) \\ (E-mail: amanzanares@ucam.edu)
}

\begin{abstract}
This research aims to test the suitability of a protocol for automated measurement to describe visual and motor behaviour in the process of learning to sail. The objective is to provide coaches with the necessary scientific and technological support to analyse the variables of success in race starting. The study was performed with a highly ranked sailor in the Optimist class ranking. The instruments used to carry out the investigation were the sailing simulator VSail-Trainer ${ }^{\circledR}$ and the eye tracking system ASL ${ }^{\circledR}$. Two simulated race starts were performed with a protocol of five minutes. The results show the automated protocol is suitable for measuring the ability of boat handling and visual performance in simulated conditions. Visual behaviour shows that the sailor visually fixates on locations that provide relevant information for the race start such as clock, other competitors, wind direction and the start buoys.
\end{abstract}
KEYWORDS
1. Boat.
2. Control.
3. Human Factor.
4. Instrument approach.

Submitted: 6 July 2015. Accepted: 28 October 2015. First published online: 20 November 2015.

1. INTRODUCTION. Sailing is a sport linked to the instability and the uncertainty of the environment. Wind direction, sea conditions and behaviour of other competitors are unpredictable and therefore generate great uncertainty among sailors. Sailing is a sport influenced by the environment (sea state, wind speed and wind direction) and the actions of other competitors (Araujo et al., 2005). Hence, sailors are constantly adjusting boat handling and taking the most appropriate decisions according to the changing conditions perceived from these elements.

Despite the difficulty of studying this sport, some authors have identified the determining performance capabilities. According to Bertrand (1993) physical fitness, boat speed and tactical intelligence are the main determinants of competitive performance in dinghy sailing. Thill (1983), argues that success is largely determined by a sailor's 
ability to adapt to environmental conditions. The boat hull design, technology applied to sailing, physiological characteristics, physical capabilities, technique, tactics, sailor mentality, injuries, nutrition and experience, among others, may affect the performance of sailors (Araujo et al., 2005; Araujo and Serpa, 1998; Manzanares et al., 2012; Spurway et al., 2007).

Many investigations seek to discover and improve the factors that affect success, although the aim of most of the efforts is to determine the most relevant physical capabilities in this sport.

Sailing is a sport that requires a high level in the perception of stimulus. At the same time, sailing requires an optimum capacity to extract meaningful information from the environment, anticipate the appearance of the main visual indices and make decisions that facilitate continuous adaptation to the constraints of the environment, thereby creating an ideal pattern of visual and motor behaviour.

During a race, sailors are constantly obtaining information from the environment through the senses. The most decisive sense in sailors is sight; since with this sense sailors perceive the stimuli present in the sailing environment that will determine their response. Through sight, the sailor is able to determine the position and actions of other competitors, sea conditions and even direction and intensity of wind gusts. There are studies that suggest sight is the most efficient channel to capture information of our surroundings while practicing sport (Magill, 1993; Reina et al., 2006). MacLeod (1991) established that sight is the most accurate receptor system to obtain information about movement and spatial and temporal characteristics around us. Sight is recognised as the main source of afferent information when high spatial precision is required (Abahnini et al., 1997), as is the case in sailing sports.

Some authors focus their research on visual and motor behaviour in athletes of different sports (e.g. Martell and Vickers, 2004; Reina et al., 2007; Wilson et al., 2009). Williams (2000) used the strategy of visual search as a tool for identifying talent in soccer players. Similarly, Wilson et al. (2009) studied the relationship between the visual fixation place of basketball players during free throw shooting situations and their performance. The research carried out by Reina et al., (2006), studied the visual behaviour and motor response of three groups of tennis players ranked by their disability characteristics and experience, in a situation of tennis serving, both real (3D) and simulated (2D). This research showed the importance of studying the visual behaviour of athletes in simulated conditions, being a truly influential factor in the performance of many sports in which environmental conditions are more stable than dinghy sailing.

In order to control the effect of environmental conditions in sport and as a preliminary step for a research in natural conditions, the analysis of motor and visual behaviour through automated systems was applied in several research areas. Afonso et al. (2012), Afonso and Mesquita (2013), Dicks et al. (2010), Loveday and Coast (2010), Reina et al. (2007) and Williams and Davids (1998) developed protocols to record motor response and visual behaviour in volleyball, soccer, cycling or tennis among others. However, in sailing no works of these characteristics are found, possibly due to the complexity of the sport when practiced in real conditions, as discussed above.

Hence, the need to design protocols that allow the recording of performance variables in this sport. A sailing simulator is a device that allows control of variables that may be difficult or impossible to control in open water. In this way, it becomes an interesting research tool that facilitates the development and optimisation of 
measurement protocols for subsequent transfer to the investigation in open water. For that reason, the aims of this research are firstly to design and apply an automated measurement protocol to simulated sailing, secondly to record, using technological devices, visual and motor behaviour related to performance variables, and finally to evaluate the usefulness of the protocol for the analysis of visual and motor performance on sailors and thier potential transference to real sailing.

\section{METHOD}

2.1. Participants. One sailor of the Optimist class. The Optimist class is an international sailing class to learn sailing and initiation in racing. The sailor was top of the Optimist class ranking with a sailing experience of 6 years, aged 12 years old (weight $49 \mathrm{~kg}$; height $155.5 \mathrm{~cm}$; arm span $158 \mathrm{~cm}$ ). The participant gave his consent to participate in the research as well as his parents or legal representatives.

2.2. Measurements. The variables analysed were: 1) visual behaviour, defined through the study of the location of gaze fixation, amount of fixations and time of fixations. A fixation can be defined as the gaze remaining stable on one location over at least 99.9 ms (3 frames) (Williams et al., 1999); 2) motor behaviour (boat handling), studied by analysing boat speed, distance covered, horizontal and vertical trajectory described by the boat and the variability of the trajectory (the latter was analysed using the coefficient of variation and sample entropy -SampEn- (Richman and Moorman, 2000), that allows the analysis of the predictability of time series data and 3) successful start of the race, defined from the assessment of the boat position at the moment of the starting signal. SampEn quantifies the regularity of time series with higher values associated with a greater irregularity. SampEn is the negative of the natural logarithm of the probability that if two sets of simultaneous data points $(N)$ of length $m$ have distance $<r$ then two sets of simultaneous data points of length $m+1$ also have distance $<r$. SampEn takes values from zero to two, with zero the highest periodicity or regularity (lowest complexity). $N$ or time series size, a length $m$ determine the size of the compared vectors and a tolerance window $r$ that tends to standardize employing the original series standard deviation (Menayo et al., 2014).

Eighteen visual locations were identified in the scene by a committee of expert coaches. Those locations were ranked by the level of relevant information they provided, with the first location the most important and the last the least relevant: 1) bow, 2) rest of the boat, 3) tack of the sail, 4) rest of the sail, 5) tell-tales, 6) wind direction, 7) clock, 8) other competitors, 9) start buoys, 10) race buoys, 11) sailor's position on the map, 12) start buoys on map, 13) race buoys on map, 14) other competitors on map, 15) others, 16) rest of scenery (where locations like the sky, the coast or the horizon are considered), 17) boom, 18) mast.

The following variables were controlled: luminosity $(40 \mathrm{~lm} / \mathrm{m} 2)$, room temperature $\left(28^{\circ} \mathrm{C}\right)$, wind speed during the simulated race $(12 \mathrm{knots}$, stable wind and not favoured start), starting sequence (six minutes), number of opponents (five) and their behaviour. All these variables remained stable from the moment the sailor entered the research room until the race ended.

In relation to the instruments, an automated system was used, integrated by a virtual sail simulator (vSail-Trainer ${ }^{\mathrm{R}}$ ), designed by the company Virtual Sailing Pyt $\mathrm{Ltd}^{\mathrm{TM}}$, one eye tracking system (MobileEye ${ }^{\mathrm{TM}}$ ) created by Applied Sciences Laboratories (ASL ${ }^{\circledR}$ ) 


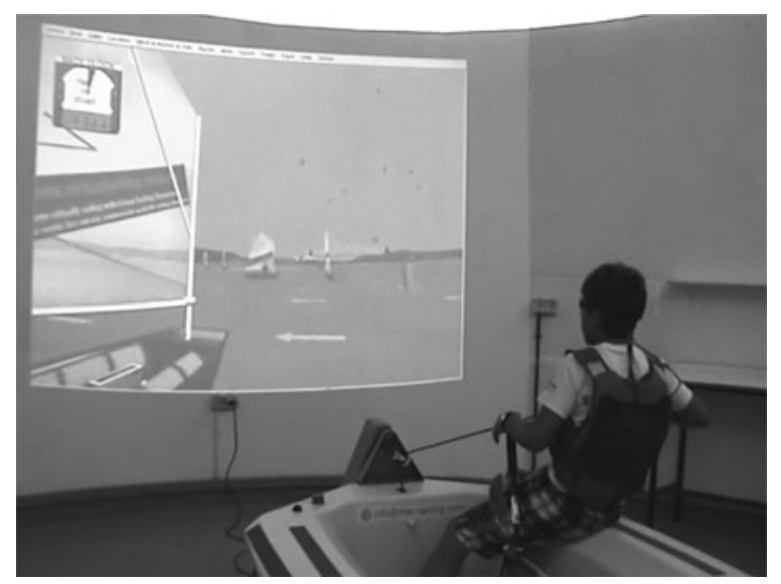

Figure 1. Subject sailing during the measurement process.

and a system of audio-visual projection integrated by a multimedia projector (Toshiba EX21) and a loudspeaker system (Logitech Z623), through which the sound of the race (wind, sea, sail movement and start signal) was reproduced.

The vSail-Trainer ${ }^{\mathbb{R}}$, designed by the company Virtual Sailing Pty Ltd, consisted of one boat hull, which had a size (length: $230 \mathrm{~cm}$, breadth: $150 \mathrm{~cm}$ ) similar to an Optimist boat (length: $230 \mathrm{~cm}$, breadth: $113 \mathrm{~cm}$ ). The sailor controlled the simulator as if it was a normal boat, the rudder was used to control the course/direction and a mainsheet to control the mainsail. Sailors have to control the heeling of the boat by changing the position of their body. The instruments cannot simulate the pitching of the boat or the depth of the keel. The simulation was screened in front of the sailor. The image size $(2.00 \times 2.50 \mathrm{~m})$ was designed to simulate a real situation (Reina et al., 2004). The virtual situation was controlled by the simulator software, which can control wind strength, gusts and sailing conditions.

The eye tracking system Mobile Eye ${ }^{\mathrm{TM}}$ is a head-mounted, monocular eye tracking system that computes point of gaze within a scene through calculation of the vector (angle and distance) between the participant's pupil and cornea. The video is relayed from the Mobile Eye ${ }^{\mathrm{TM}}$ scene-mounted unit to a remote unit attached to a modified digital video cassette recorder (DVCR, Sony GV-12 D1000E). Gaze behaviour data were collected at a rate of 30 frames per second $(30 \mathrm{~Hz})$ and subjected to a frame-by-frame analysis.

2.3. Procedure. Data collection was carried out in a circular room, $6.48 \mathrm{~m}$ diameter and $4.00 \mathrm{~m}$ high. The simulation was located in the centre of the room at an equal distance from the walls.

After the sailor came into the research room, measures of weight, height and arm span were performed. Following these anthropometric measurements, the sailor took a seat in front of the play screen where a video presentation was screened, showing the simulated sailing situation, the simulator controls, the software and the eye tracking system function. The measurement protocol consisted of two race starts, of five minutes duration each, and a rest period of three minutes between them (Figure 1). 
After the presentation video, the sailor wore the eye tracking system with a life jacket, which contained the storage unit for this device. Immediately after the calibration of the eye tracking system was performed, calibration of the values of the mainsheet, rudder and heel of the simulator was conducted. Finally the sailor's weight, weather conditions and other competitors were introduced into the software of the simulator. Once the calibration was completed, the starting sequence began. It was divided into two phases of three minutes each. In the first part the sailor could freely use the simulator without any indication. In the second, the sailor had to navigate following guidelines previously recorded and reproduced audio-visually. After six minutes of the starting sequence, the process of measurement began.

During the race start, the sailor competed with five competitors of different levels of expertise. The time it takes them to cross the starting line, after the start signal, determines the level. The start was performed in similar conditions to the real race start, where the starting line was demarcated by buoys.

2.4. Statistical Analysis. The statistical analysis used was composed by a descriptive analysis of the variables of visual behaviour and variables of boat speed and course distance. The statistical program used for this analysis was the IBM SPSS Statistics v. $21^{\circledR}$.

The coefficient of variation of the track record in East and North directions (X and Y) was calculated from the results of boat handling. Along with these data, sample entropy (SampEn) was extracted from the track record by the boat, using software Entropy Calculation Methods (Menayo et al., 2014).

\section{RESULTS}

3.1. Motor behaviour. Figure 2 shows the trajectory described by the boat during the last minute previous to the start signal, in the first and second race. It is observed that a greater distance was travelled in the second race with respect to the first $(94 \mathrm{~m}$ compared to $196 \mathrm{~m}$ ). The average speed of the boat during the first race was $5.62 \mathrm{~km} / \mathrm{h}$, while in the second one it was $11.74 \mathrm{~km} / \mathrm{h}$. At the same time it was observed that in the second race, the sailor was closer to the start line, at the instant of the start signal, compared to the first race, since in the first one he was at $4.75 \mathrm{~m}$ from the start line compared to $2.00 \mathrm{~m}$ in the second. It should also be appreciated that the second start was performed closer to the port buoy. Finally, the speed reached by the boat at the start time was identical in both races $(8.57 \mathrm{~km} / \mathrm{h})$.

Scores obtained from sample entropy from the trajectory of the boat (Table 1) indicate that the trajectory record becomes more predictable during the second race in the East direction (-X axis-). However, in the North direction (-Y axis-), the trajectory of the boat becomes more unpredictable. The coefficients of variation of the trajectory in the East and North axes during the first race, were $28 \cdot 31 \%$ and $18 \cdot 34 \%$, respectively, while in the second, were $83.94 \%$ in the East axis and $40.67 \%$ in the North axis. These results show a difference between the variability of the trajectory of the second start with respect to the first.

3.2. Visual behaviour. Figure 3 shows the number and percentage of fixations performed on each location at where the sailor fixed his gaze over last minute of the course previous to the first and second start signal. The number of fixations performed in the first race was 94 , while in the second race, 86 fixations were performed. 


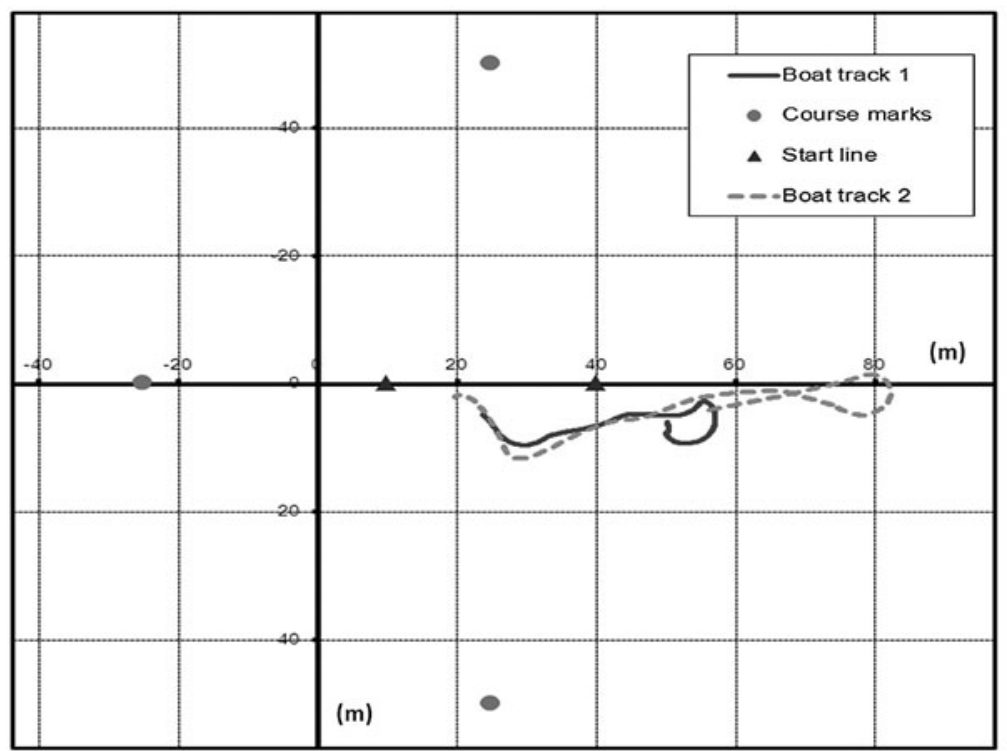

Figure 2. Boat track on the 1st and 2nd start.

Table 1. Sample entropy and coefficient of variation of the boat track on East and North trajectory $(\mathrm{X}=$ East displacement; and $\mathrm{Y}=$ North displacement).

\begin{tabular}{lccr}
\hline & $1^{\text {st }}$ Start & $2^{\text {nd }}$ Start & Contrast \\
\hline Sample entropy on X axis & 0,070 & 0,058 & 0,012 \\
Sample entropy on Y axis & 0,011 & 0,015 & $-0,004$ \\
Coefficients of variation on X axis & $28,31 \%$ & $83,94 \%$ & $-55,63$ \\
Coefficients of variation on Y axis & $18,34 \%$ & $40,67 \%$ & $-22,33$ \\
\hline
\end{tabular}

Figure 4 shows the average time that the sailor was fixing his gaze at each location and the percentage of time fixations. The average fixation times of the first and second race were 0.42 and 0.49 seconds respectively.

According to Figures 2 and 3, during the first start, the sailor performed a greater number of fixations in locations like "bow", "wind direction", and "tell-tales". Furthermore, the fixation time in the same locations was lower in the second start than in the first one. In contrast, on the second start, the sailor performed a greater number of fixations with a lower time of fixations at the location "other competitors".

Moreover, there are locations where differences in the number of fixations between the two starts can be observed, as in "tack of the sail", "wind direction" "start buoys", "rest of the boat" and "other competitors on map". It is also notable that the increase in the percentage of fixation time on location "sailor's position on the map" in the second race $(13 \cdot 4 \%)$ compared to the first one $(5 \cdot 8 \%)$.

4. DISCUSSION. The aim of this study was to develop an automated measurement protocol to analyse visual behaviour and ability to handle boat to find out if those factors could be related to success in the race start. 


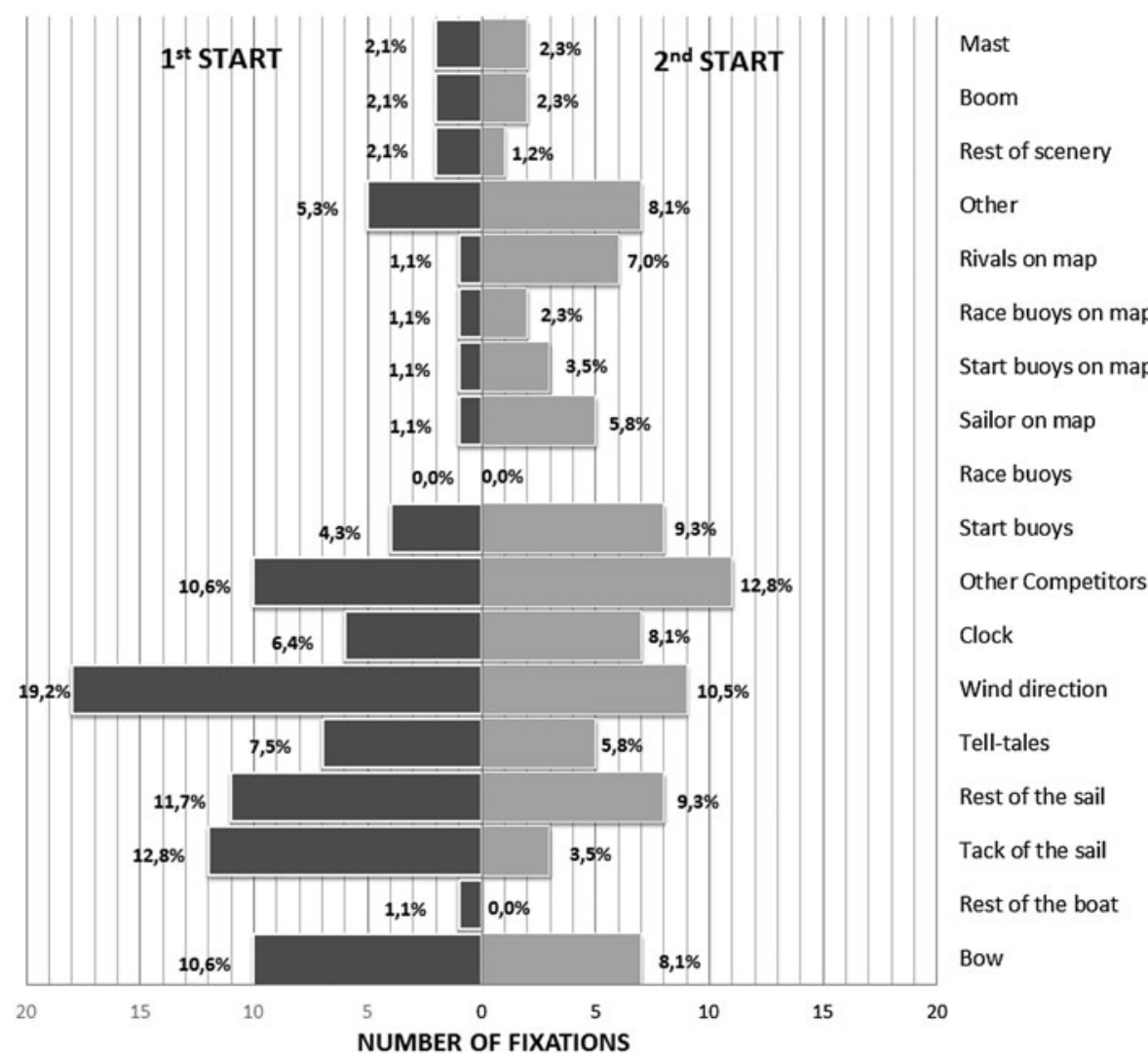

Figure 3. Quantity and percentage of fixations.

4.1. Usefulness and application of the measurement protocol. This automated measurement protocol is appropriate for the recording and analysis of the motor and visual behaviour of the sailor during a simulated race start. All devices were integrated, facilitating the work of the researcher during data collection. The suitability of the system to investigate in a sailing simulator is evident, providing a clear view to transfer the protocol to real sailing situations. This protocol will allow scientific support to learn and train using simulators. It was shown that visual and motor analysis in this simulated environment is viable, and can be a useful tool to introduce the learner to sailing in real environments. The simulator allows starting the learning processes in a controlled situation, being able to determine the initial conditions of practice and performance. The variables selected for analysis appear appropriate, combining perceptual and motor behaviour, as performance factors of sailors.

4.2. Motor behaviour. The protocol developed allows the measurement of handling capacity of the boat by the sailor, through the registration of the trajectory described in the race. The results of the ability to handle the boat, extracted from the analysis of trajectory of the boat during the last minute of the race start, suggest a trend towards increasing the handling boat capability. This trend is identified because in the same time of sailing, the sailor toured a greater distance, leading to 


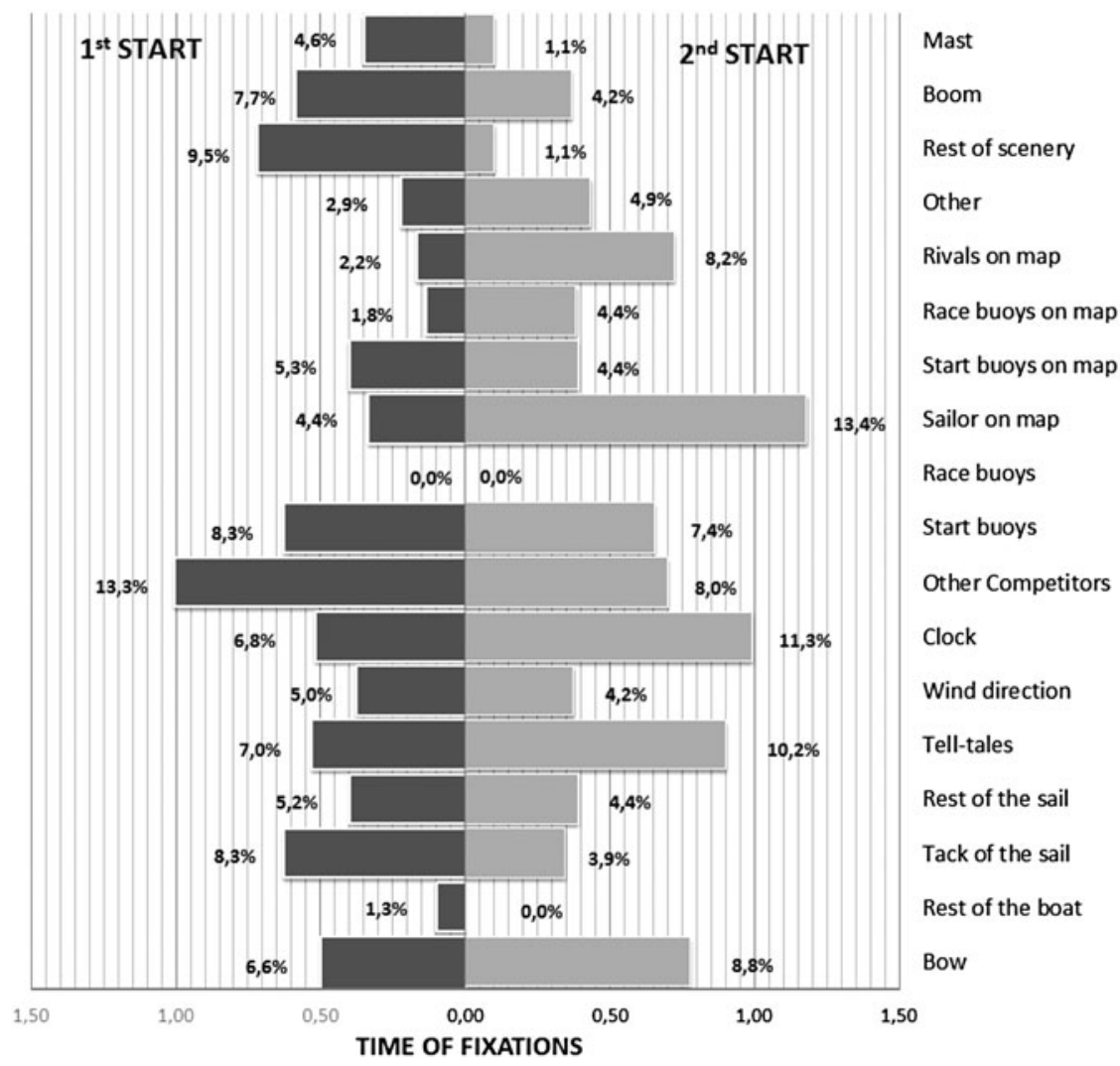

Figure 4. Time of fixations and percentage in relation to the total fixation time.

an increase in the average speed of the boat. Furthermore, in the second start, at the moment of the start signal, the sailor is closer to the start line compared to the first race. This is an indicator of an increase in success.

Comparing the two outputs, it is seen that the trajectory of the boat becomes more predictable in East displacement (X axis), while in the North direction (Y axis) it tends to be unpredictable, although the difference is minimal. This behaviour shows an increased performance due to the practice in the first race, because in the second race, the boat performed a less complex trajectory in the East direction. In spite of the fact that the vertical trajectory tends to be less predictable, it may be an element that determines a functional or exploratory behaviour to find the best route in this direction. The above-mentioned results are in accordance with the review of several studies, as performed by Bartlett (2008) about the existence of functional variability in different sports action. Similarly, Kudo and Othsuki (2008) affirm that functional variability in different sports actions could be decisive in sport learning.

Regarding the results of variability of the trajectory provided by the coefficients of variation, the increase in this parameter in both directions ( $\mathrm{X}$ and $\mathrm{Y})$ in the second race with regards to the first one is striking. This result would support that as previously discussed, an increased amount of variability in the trajectory of the boat also 
means a greater yield in terms of handling capacity of the boat. In this sense, these results are not unusual, because there are studies with skilled athletes in which skills such as kinematics and kinetics of launching a baseball or basketball free throw were analysed, concluding that there are no reductions in the variability arising from the expert status (Fleisig et al., 2009; Button et al., 2003).

4.3. Visual behaviour. The protocol developed allows the integration and registration of sailor visual behaviour with the ability to handle the boat in real time. When analysing the results of visual behaviour, it appears that there are differences in the total number of fixations between the first and the second start. The results agree with the results reported by Avila and Moreno (2003), who claimed that the strategy of visual search is more effective when the athlete performs a reduced number and longer lasting visual fixations.

Similarly, the location with the highest number of fixations during the first race is "wind direction", considering this behaviour as ineffective, while the localisation with the higher number of fixations in the second race is "other competitors". This behaviour is closer to an expert visual behaviour pattern. The expert visual search strategy tends to perform a small number of fixations, with increased fixation time, as happens in the second start (Moreno et al., 2005; Reina et al., 2007).

As Seung-Min (2010) demonstrated with volleyball players, novice players use a less active strategy of visual search and are more focused on specific locations, while expert players use a more active strategy, obtaining information from locations that novices did not consider as important or performing a more homogeneous distribution of the fixations among the locations. The results of visual performance of the sailor shows a more homogenous distribution of fixations among total fixations during the second race start, compared to the first one. Similarly, during the second start, the number of fixations on most locations decreased, making a visual search strategy more active than during the first start.

Finally, the increase in the percentage of fixation time on location "sailor's position on the map" in the second race could be identified with a possible visual point (Savelsbergh et al., 2002; Reina et al., 2007). This location corresponds to an area that does not provide relevant visual information independently. In this sense, Williams and Davids (1998) suggested that athletes use peripheral vision in complex environments, peripheral vision being more sensitive to movement than fovea vision. In this case, the sailor would extract relevant information from nearby areas, without moving his eyes. Thus, the sailor would be developing a more optimal visual strategy in the second race.

For the sailor, a simulated race situation is a new environment that has not been tested before. For this reason, we must consider that as reported in Williams et al. (1999), in sports such as hockey, expert athletes have a similar number of fixations in real and simulated situations. On the contrary, it may happen that there is not a similarity in the number of fixations that are performed in a real situation and in a simulated one, as in the investigation of Reina et al. (2006) in tennis players. However, we do not have the right material to prove this hypothesis, since the device used for visual search investigation cannot currently be subjected to wet conditions.

5. CONCLUSION. Visual behaviour developed by the sailor over the course of both simulated starts shows a trend towards improved visual search strategy, acquiring 
a visual behaviour nearer to expert. In spite of the changes between the first and the second start, it is noteworthy that during both races, the sailor made a greater number of fixations on the bow of the boat, clock, mainsail, other competitors, wind direction and start buoys. These locations are presupposed very important to obtain relevant information about the course of the race.

Similarly, there was an improvement in the control of the boat in the second start, because the sailor was closer to the start line, with the aim of being as close as possible, without crossing it.

In conclusion, during the second race the sailor obtained more successful results in visual and motor behaviour. These improvements may be due to practice that the sailor acquired during the starting sequence and the first race. In this sense we can say that the proposed protocol on sailing simulation could help to improve learning and performance of sailors.

The first limitation of this investigation is the sample size; because only one sailor was evaluated and the results obtained by this sailor cannot be extrapolated to other sailors from the same class. The second limitation is the lack of real wind; this is the great difference between real and simulated situation, although the lack of real wind can be an advantage, because it allows control of the learning and training process.

The third limitation is the effect of competitors, since the simulator allows control of the level of the rivals but does not permit comparison of their positions with the position of the sailor. The competitors were included in order to provide realism to the protocol.

Finally, it must be pointed out that it would be necessary to continue the study of visual and motor behaviour of sailors in different boat classes, with the aim of defining the suitable pattern of visual and motor behaviour to achieve sport success, as well as increasing the number of participants in order to generalise the obtained results and assess the transfer of study to real situations.

\section{REFERENCES}

Abahnini, K., Proteau, L. and Temprado, J.J. (1997). Evidence supporting the importance of peripheral visual information for the directional control of aiming movement. Journal of Motor Behavior, 29, 230-242.

Afonso, J. and Mesquita, I. (2013). Skill-based differences in visual search behaviours and verbal reports in a representative film-based task in volleyball. International Journal of Performance Analysis in Sport, 13, 669-677.

Afonso, J., Garganta, J., McRobert, A., Williams, A.M. and Mesquita, I. (2012). The perceptual cognitive processes underpinning skilled performance in volleyball: Evidence from eye-movements and verbal reports of thinking involving an insitu representative task. Journal of Sports Science and Medicine, 11, 339-345.

Araujo, D., Davids, K. and Serpa, S. (2005). An ecological approach to expertise effects in decision-making in simulated sailing regatta. Psychology of Sport and Exercise, 6, 671-692.

Araujo, D. and Serpa, S. (1998). Toma de decisión dinámica en diferentes niveles de expertise en el deporte de vela. Revista de Psicología del Deporte, 8, 103-115.

Avila, F. and Moreno, F.J. (2003). Visual search strategies elaborated by tennis coaches during execution error detection process. Journal of Human Movement Studies, 44, 209-224.

Bartlett, R.M. (2008). Movement variability and its implications for sports scientists and practitioners: an overview. International Journal of Sports Science \& Coaching, 1(3), 113-124.

Bertrand, L. (1993). Australian Yachting Federation elite training program - sports science quadrennial plan 1993-1996. Australian Yachting Federation.

Button, C., Macleod, M., Sanders, R. and Coleman, S. (2003). Examining movement variability in the basketball free-throw action at different skill levels. Research Quarterly for Exercise and Sport, 14, 257-269. 
Dicks, M., Button, C. and Davids, K. (2010). Examination of gaze behaviors under in situ and video simulation task constraints reveals differences in information pickup for perception and action. Attention, Perception and Psychophysics, 72, 706-720.

Fleisig, G., Chu, Y.C., Weber, A. and Andrews, J. (2009) Variability in baseball pitching biomechanics among various levels of competition. Sports Biomechanics, 8, 10-21.

Kudo, K. and Ohtsuki, T. (2008). Adaptative variability in skilled human movements. Information and Media Technologies, 3, 409-420.

Loveday, D.E. and Coast, J.R. (2010). Simulated time trial using three different pedal rate protocols. International Journal of Performance Analysis in Sport, 10, 25-32.

Magill, R. A. (1993). Motor learning. Concepts and applications. Iowa: Brown Publishers.

Manzanares, A., Segado, F. and Menayo, R. (2012). Determinants factors on performance the practice of sailing: literature review. Cultura, Ciencia y Deporte, 20, 125-134.

Martell, S. and Vickers, J.N. (2004). Gaze characteristics of elite and near-elite ice hockey players. Human Movement Science, 22, 689-712.

McLeod, B. (1991). Effects of eyerobics visual skill training on the selected performance measures of female varsity soccer players. Perceptual and Motor Skills, 72, 863-866.

Menayo, R., Encarnación, A., Gea, G.M. and Marcos, P.J. (2014). Sample entropy-based analysis of differential and traditional training effects on dynamic balance in healthy people. Journal of Motor Behavior, 46, 73-82.

Moreno, F.J., Luis, V., Salgado, F., García, J.A. and Reina, R. (2005).Visual behavior and perception of trajectories of moving objects with visual occlusion. Perceptual and Motor Skills, 101, 13-20.

Reina, R., Moreno, F.J. and Sanz, D. (2007). Visual behavior and motor responses of novice and experienced wheelchair tennis players relative to the service return. Adapted Physical Activity Quarterly, 24, 254-271.

Reina, R., Moreno, F.J., Sanz, D., Damas, J.S. and Luis, V. (2006). El efecto de la dimensionalidad de la escena en el comportamiento visual y motor durante el resto al servicio en tenis y tenis en silla de ruedas. European Journal of Human Movement, 16, 63-84.

Reina, R., Del Campo, V.L., Moreno, F.J. and Sanz, D. (2004). Influencia del tamaño de la imagen sobre las estrategias de búsqueda visual en situación simulada del resto de tenis. Revista de Psicología del Deporte, 13, 175-193.

Richman, J.S. and Moorman, J.R. (2000). Physiological Time-Series Analysis using Approximate Entropy and Sample Entropy. American Journal of Physiology. Heart and Circulatory Physiology, 278, 2039-2049.

Savelsbergh, G., Williams, A.M., Van der Kamp, J. and Ward, P. (2002). Visual Search, anticipation and expertise in soccer goalkeepers. Journal of Sports Sciences, 20, 279-287.

Seung-Min, L. (2010). Does your eye keep on the Ball?: The Strategy of Eye Movement for Volleyball Defensive Players During Spike Serve Reception. International Journal of Applied Sports Sciences, 22, $128-137$.

Spurway, N., Legg, S. and Hale, T. (2007). Sailing Physiology. Journal of Sports Science, 25, 1073-1075.

Thill, E. (1983). La detection sportive á partir des crotéres psychologiques. En Riox, G. \& Thill, E. (Ed.), Competition sportive et psychologie. Journées Européenes de Psychologie du Sport (pp. 77-94). Paris: Chiron.

Williams, A.M. (2000). Perceptual skill in soccer: Implications for talent identification and development. Journal of Sports Sciences, 18, 737-750.

Williams, A.M. and Davids, K. (1998). Visual search strategy, selective attention and expertise in soccer. Research Quarterly for Exercise and Sport, 69, 111-129.

Williams, A.M., Davids, K. and Williams, J.G. (1999). Visual perception and action in sports. London: E \& FN Spon.

Williams, A.M., Swarbrick, L.C., Grant, A. and Weigelt, C. (1999). Visual search strategy, recall ability, and expertise in field hockey. Journal of Sport and Exercise Psychology, 21, S123.

Wilson, M.R., Vine, S.J. and Wood, G. (2009). The influence of anxiety on visual attentional control in basketball free throw shooting. Journal of Sport \& Exercise Psychology, 31, 152-168. 